DOI: $10.23857 / p c . v 3 i 7.566$

Recepción: 18 / 04 / 2018

Aceptación: 16 / 06 / 2018

Publicación: 02 / 07 / 2018

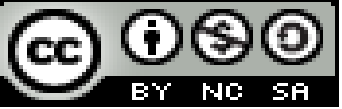

Ciencias económicas y administrativas

Artículo de investigación

\title{
Análisis estadístico descriptivo de los indicadores políticos que potencian el turismo en Riobamba
}

\section{Descriptive statistical analysis of the political indicators that promote tourism in Riobamba}

\section{Análise estatística descritiva dos indicadores políticos que promovem o turismo em Riobamba}

\author{
Catalina M. Verdugo-Bernal ${ }^{\mathrm{I}}$ \\ cverdugo@espoch.edu.ec \\ Ana C. Flores-Mancheno II \\ acflores@espoch.edu.ec \\ Sandra P. Miranda-Salazar ${ }^{\text {III }}$ \\ spmiranda@espoch.edu.ec \\ Cecilia T. Limaico-Nieto IV \\ cecilia.limaico@espoch.edu.ec
}

Correspondencia: cverdugo@espoch.edu.ec

\footnotetext{
${ }^{I}$ Magister en Turismo Sostenible y Desarrollo Local, Master en Diseño, Gestión y Dirección de Proyectos, Especialista Superior en Gestión Ambiental Mención Amazonia y Derechos Humanos, Ingeniera en Ecoturismo, Docente en la Escuela Superior Politécnica de Chimborazo, Riobamba, Ecuador.

${ }^{\text {II }}$ Master Universitario en Estadística Aplicada, Ingeniera en Estadística Informática, Docente de la Escuela Superior Politécnica de Chimborazo, Riobamba, Ecuador.

${ }^{\text {III }}$ Magister en Ciencias Sociales con Mención en Desarrollo Local y Territorial, Ingeniera en Ecoturismo, Docente en la Escuela Superior Politécnica de Chimborazo, Riobamba, Ecuador.

${ }^{\text {IV }}$ Magister en Educación Matemática, Doctora en Física, Licenciada en Ciencias de la Educación Profesora de Enseñanza Media en la Especialización de Matemática y Física, Docente en la Escuela Superior Politécnica de Chimborazo, Riobamba, Ecuador.
} 


\title{
Resumen
}

La sostenibilidad de las políticas turísticas podría ser un factor decisivo en la mejora de la economía local como meta gubernamental y parte fundamental de la sostenibilidad integral de la actividad turística. El problema que enfrenta la ciudad de Riobamba es la escasa información y un débil sistema de indicadores referente a políticas públicas turísticas y patrimoniales, por ello se propone identificar una serie de variables que contribuyan a generar sostenibilidad socio-económica en la ciudad. El trabajo propuesto se realizó a través del análisis de variables específicas como la concepción de política turística, cultural, patrimonial; la situación del turismo y el patrimonio en la ciudad; las relaciones y afectaciones entre los actores sociales, proyección y perspectivas de las políticas turísticas y patrimoniales existentes; fomentando el análisis, la opinión y la participación de diferentes involucrados los cuales se constituyen en actores sociales, escenarios turísticos, posibles metas y prioridades con las necesidades reales del desarrollo del turismo en Riobamba. Amparados en estas premisas, la Escuela Superior Politécnica de Chimborazo y el Gobierno autónomo descentralizado de Riobamba desarrollan un proyecto de medición de indicadores que inciden en la gestión turística de Riobamba, con la participación conjunta de estudiantes, docentes, investigadores y técnicos en la ardua tarea de levantar una línea base de información sobre diferentes ámbitos de la sostenibilidad para descubrir los factores que intervienen en la sostenibilidad urbana del turismo a través de una análisis estadístico descriptico y poder analizar esta dinámica propiciando una serie de recomendaciones para las instituciones involucradas.

Palabras clave: Análisis estadístico; indicadores; político-institucional; turismo; Riobamba.

\begin{abstract}
The sustainability of tourism policies could be a decisive factor in the improvement of the local economy as a governmental goal and a fundamental part of the integral sustainability of tourism activity. The problem faced by Riobamba is the scarce information and a weak system of indicators referring to tourism and heritage public policies, for this reason it is proposed to identify a series of variables that contribute to generate socio-economic sustainability in the city. The proposed work was carried out through the analysis of specific variables such as the conception of tourism, cultural, patrimonial policy; the situation of tourism and heritage in the city; the relationships and effects among the social actors, projection and perspectives of existing tourism and heritage
\end{abstract}


policies; fostering the analysis, opinion and participation of different stakeholders which constitute social actors, tourist scenarios, possible goals and priorities with the real needs of tourism development in Riobamba. Sheltered in these premises, Escuela Superior Politécnica de Chimborazo (ESPOCH) and Decentralized autonomous government of Riobamba (GAD) develop a project to measure indicators that affect the tourist management of Riobamba, with the joint participation of students, teachers, researchers and technicians in the arduous task to build a baseline of information on different areas of sustainability to discover the factors that intervene in the urban sustainability of tourism through a descriptive statistical analysis and to analyze this dynamic by providing a series of recommendations for the institutions involved.

Keywords: Statistical analysis; indicators; political-institutional; tourism; Riobamba.

\section{Resumo}

A sustentabilidade das políticas de turismo pode ser um fator decisivo para a melhoria da economia local como meta governamental e parte fundamental da sustentabilidade integral da atividade turística. O problema de frente para a cidade de Riobamba é a falta de informação e de um sistema fraco de indicadores relativos a turismo e políticas públicas herança, portanto, propõe-se a identificar uma série de variáveis que contribuem para a geração de sustentabilidade sócioeconômico da cidade. O trabalho proposto foi realizado por meio da análise de variáveis específicas, como a concepção de política turística, cultural, patrimonial; a situação do turismo e do patrimônio na cidade; as relações e efeitos entre os atores sociais, projeção e perspectivas das políticas existentes de turismo e patrimônio; fomentar a análise, opinião e participação de diferentes atores que constituem atores sociais, cenários turísticos, possíveis metas e prioridades com as necessidades reais do desenvolvimento do turismo em Riobamba. Coberto nestas premissas, a Escola Politécnica de Chimborazo eo Governo descentralizado Riobamba desenvolver um projecto de medida indicadores que afetam a gestão do turismo de Riobamba, com a participação conjunta de estudantes, professores, pesquisadores e técnicos na tarefa árdua construir uma linha de base de informações sobre diferentes áreas de sustentabilidade para descobrir os fatores que intervêm na sustentabilidade urbana do turismo por meio de uma análise estatística descritiva e analisar essa dinâmica, fornecendo uma série de recomendações para as instituições envolvidas.

Palavras chave: Análise estatística; indicadores; político-institucionais; turismo; Riobamba. 


\section{Introducción}

Los indicadores sin duda, constituyen una de las herramientas indispensables para evaluar y predecir tendencias de la situación de una región o localidad en cuanto a la toma de decisiones en cuestiones económicas, sociales y políticos, así como para valorar el cumplimiento de las metas y objetivos fijados en la planificación gubernamental (López, \& Gentile, 2008, p.1).

Los indicadores políticos en el ámbito turístico inspeccionan el comportamiento de la gestión gubernamental de la actividad turística en relación a los factores gobierno-ciudadanía. (Gomis, et. At.2006, pp.1-2), además, sirven de complemento a los populares sistemas de información geográfica para facilitar la labor de los gestores turísticos en lo referente a la planificación y creación de políticas turísticas relacionadas con la sostenibilidad. (García,2014). En Ecuador, se organiza la gestión turística a través de su Ministerio de Turismo el que genera y da seguimiento a las políticas y al desempeño institucional. (Pérez, 2002. pp.52-54).

A partir de esos indicadores, es posible generar un conjunto de información sobre la situación real del desarrollo turístico, en lo que se refiere a los aspectos sustentables e insustentables, lo cual deberá subsidiar la toma de decisiones de los diversos actores involucrados en su planificación para que la definición de las acciones futuras a ser adoptadas, estén basadas en prácticas sustentables. (De Oliveira, et. al,p. 181)

Las políticas públicas turísticas sostenibles dentro de la planificación son factores esenciales que permiten la formulación de proyectos y el desarrollo armónico de la actividad turística así obteniendo beneficios económicos, sociales para la comunidad. (Díaz, 2003). Este ciclo de desarrollo propicia una estructura en la cual la toma de decisiones colectivas e individuales es eficiente y eficaz logrando la sostenibilidad del lugar. (Goeldner, et. al.2002, p. 294). El rol de las mismas, así como su implicancia, varían conforme las necesidades y los objetivos de cada lugar.

En este panorama, identificar indicadores políticos que coadyuvan a la sostenibilidad urbana en diferentes áreas y países, particularmente en el tema turístico facilitan el manejo y crecimiento territorial, puesto que promueven metodologías y herramientas de trabajo que además de describir la realidad de manera independiente potencian el desarrollo local (Gallopín, 2006, p. 8-10). 
Donde el Diseño del Plan Estratégico de Desarrollo de Turismo Sostenible para Ecuador (PLANDETUR 2020), busca guiar acciones a través de metas comunes y objetivos específicos, con el involucramiento activo de entidades gubernamentales, actores públicos bajo la rectoría del Ministerio de Turismo (MINTUR), privado y comunitario, a través de las cámaras de turismo, los gremios y las comunidades locales. (Equipo Consultor Tourism \& Leisure-Europraxis 2007).

En este contexto, el desarrollo de esta investigación permitirá obtener información clave para la gestión en el ámbito turístico y se enfocó en determinar indicadores que midan la Gestión Política Municipal dentro del turismo, para contribuir a potenciar la sostenibilidad integral de la ciudad con la finalidad de realizar una clasificación del nivel de calidad alcanzada por el GADMR, a partir de la valoración de variables que reporten datos confiables, los cuales fueron obtenidos a través de encuestas efectuadas a la ciudadanía e instalaciones de alojamiento y gastronomía, considerando todos los posibles procesos agregados de valor, lo cual permitió identificar las potencialidades y vulnerabilidades de la gestión política que ha realizado el municipio en el área urbana de Riobamba.

\section{Metodología}

El estudio propuesto es de tipo descriptivo, transversal, se analizan variables de tipo cualitativo y cuantitativo. Se recopilaron datos de fuentes secundarias o bibliográficas y primarias en una investigación participativa de campo proporcionada por actores sociales relevantes, estos actores fueron determinados mediante el cálculo de la muestra poblacional. Los objetivos se cumplieron de la siguiente manera:

\section{Construir un instrumento para el análisis de políticas turísticas locales a ser aplicadas a actores relevantes en las parroquias urbanas de Riobamba.}

\section{- Determinación de Población y Muestra}

Se trabajó con dos poblaciones, la primera constituida por habitantes de la ciudad, la segunda por establecimientos de alojamiento y alimentación; ambos grupos se contrastaron para medir el conocimiento de las políticas turísticas en la ciudad.

La muestra dela ciudadanía riobambeña y la muestra de establecimientos turísticos se determinaron aplicando la fórmula de población finita. 


$$
\begin{gathered}
n=\frac{N \times p \times q \times Z^{2}}{e^{2}(N-1)+p \times q \times Z^{2}} \\
n=\frac{225741 \times 0.5 p \times 0.5 \times 1.96^{2}}{0.05^{2}(225741-1)+0.5 \times 0.5 \times 1.96^{2}}
\end{gathered}
$$

\section{$n=383$ encuestas}

Donde:

$\mathrm{N}=$ número de establecimientos de servicios turísticos/población riobambeña

$\mathrm{p}=$ proporción esperada que cumple la característica deseada $(0,5)$

$\mathrm{q}=$ proporción esperada que no cumple la característica deseada $(0,5)$

$\mathrm{e}=$ Margen de error deseado $(5 \%)$

$\mathrm{Z}=1.96$

El criterio de elegibilidad para los habitantes de la ciudad será personas de ambos sexos mayores de 18 años que vivan en las 5 parroquias urbanas de Riobamba. La población de establecimientos de alojamiento y gastronomía es de $61 \%$. Finalmente, estas encuestas aplicadas a la muestra calculada para poblaciones finitas (Arboleda, 1998), son tabuladas para realizar su análisis estadístico descriptivo.

- Etapa de diseño del instrumento de recolección de información

El cuestionario traduce variables empíricas sobre las que se desea información, en preguntas concretas capaces de suscitar respuestas fiables y cuantificables. Se elaboró preguntas estructuradas y de respuesta múltiple. Se elaboró dos cuestionarios; uno para la ciudadanía de las parroquias urbanas de Riobamba con 19 preguntas, y otro para las instalaciones de alojamiento y alimentación con 25 preguntas. Con el fin de saber cuán informados se encuentran los pobladores de lo que realiza el municipio de Riobamba sobre políticas públicas en el ámbito turístico en favor del bien común.

\section{Analizar la información obtenida y su incidencia en la actividad turística de la ciudad.}


Después de recolectar la información a través de una encuesta apoyada por dos cuestionarios de tipo abanico, se procedió a la interpretación y comparación de cada uno de los ítems.

Se tabularon las variables de las dos encuestas, fue necesario la depuración de datos para asegurar un margen de error mínimo, una vez depurados los cuestionarios se procede a la codificación de las preguntas para el tratamiento informático, que consiste en el agrupamiento de datos que permitan clasificar las respuestas por variables (Alzate, 2011, párr.9).

Se asignaron códigos de respuesta asignados a cada variable para construir la base de datos que refleja la información obtenida. En lo que respecta a los procedimientos matemáticos, se utilizó una distribución de frecuencias, la misma que representa un conjunto de datos ordenados.

Para representar de manera general, en forma gráfica, el análisis porcentual de los resultados obtenidos; se emplean barras y diagramas circulares. La técnica que se utilizó, se basó en el cálculo porcentual de cada ítem.

- Prueba de proporciones de una muestra

La metodología de prueba depende de si el número de observaciones de la muestra es grande o pequeño. Esta prueba comprende el cálculo del valor estadístico de prueba $\mathrm{Z}$.

\section{Donde:}

$$
Z_{o}=\frac{\hat{p}-p_{o}}{\sqrt{\frac{p_{o} q_{o}}{n}}}
$$

$\hat{p}=x n$

$x=$ Ocurrencias

$n=$ Observaciones o tamaño de la muestra

$\hat{p}=$ Proporción de la muestra

$p o=$ Proporción hipotética o proporción propuesta

$q o=1-p o$

3. Identificación de indicadores relevantes para el turismo de Riobamba en contraste con otras investigaciones. 
Los sistemas de indicadores son una herramienta a través de la cual se puede hacer un seguimiento y evaluación de un estado y tendencia de una ciudad, es un instrumento que permite guiar futuros procesos de desarrollo urbano sectorial de la ciudad. Se analizaron fuentes secundarias de literatura relacionada a indicadores políticos en el ámbito turístico, considerados determinantes en los niveles de desarrollo sostenible en algunos países del mundo.

Además, se revisó programas y proyectos elaborados por el municipio en las parroquias urbanas de Riobamba, incluyendo su seguimiento y evaluación. Para identificar los indicadores se diseñó una ficha con: el Código del indicador, Ámbito, Indicador, Objetivo del Indicador, Fórmula, de cálculo, Interpretación, Fuente de información, Elaborado por.

La clasificación de los indicadores, son de base para una fase posterior, donde se efectuó de acuerdo al grado de impacto que generarán en el diario vivir de las personas de la Ciudad.

Se contó con la ayuda del departamento de la Dirección de Turismo del GAD Riobamba, el que contribuyó con información necesaria para la construcción de indicadores, el mismo que ayudó a la creación de una serie de propuestas para una correcta toma de decisiones referente a políticas turísticas y patrimoniales.

\section{Elaboración de un boletín informativo que permita difundir los aspectos más relevantes del trabajo realizado.}

Para la elaboración del boletín con información relacionada a indicadores de sostenibilidad políticos en el ámbito turístico de las cinco parroquias urbanas de Riobamba, se determinó que las siguientes partes son los más eficaces para la creación del mismo: Según Zúñiga, (2004, párr. 520) consta de lo siguiente: Placa del fabricante que generalmente posee un nombre corto y slogan que llama la atención del tipo de audiencia dirigida, Tabla de contenidos que es un compilado de la estructura del boletín que comunica el nombre del artículo y el número de la página donde se encuentra; Cuerpo que suele organizarse en forma de una pirámide invertida, es decir, las ideas principales en las primeras líneas o párrafos y las ideas secundarias de último con el propósito de informar los detalles más importantes del artículo desde el inicio.

\section{Resultados}




\section{A. Análisis de los resultados de campo sobre variables políticas en el ámbito turístico y su incidencia en la actividad turística de la ciudad.}

A continuación, se detallan ejemplos de cómo se tabularon las preguntas del cuestionario aplicado, que por su extensión no es posible presentar en su totalidad. Más adelante se presenta el cuadro resumen de todas las variables analizadas.

\section{Encuestas aplicadas a la ciudadanía}

Pregunta 1: Sexo de los encuestados

Tabla 1: Sexo de los encuestados

\begin{tabular}{|l|l|l|}
\hline Sexo & Frecuencia & Porcentaje \\
\hline Hombre & 213 & 54,9 \\
\hline Mujer & 162 & 41,8 \\
\hline No contesta & 13 & 3,3 \\
\hline Total & 388 & 100,0 \\
\hline
\end{tabular}

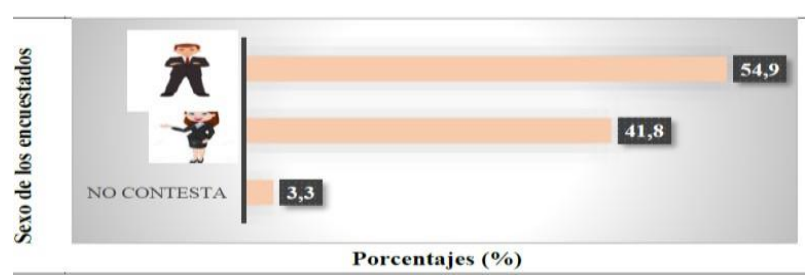

Fig. 1: Sexo de los encuestados. Moreno E, 2017

Interpretación: La participación masculina del 54,9\%, mientras que la contribución femenina al estudio fue del $41,8 \%$, el porcentaje restante de 3,3\% no contestó.

Pregunta 2: Nivel de instrucción de los encuestados

Tabla 2: Nivel de instrucción de los encuestados

\begin{tabular}{|l|l|l|}
\hline Nivel de instrucción & Frecuencia & Porcentaje \\
\hline Bachillerato incompleto & 1 & 0,3 \\
\hline Básica incompleta & 1 & 0,3 \\
\hline No contesta & 33 & 8,5 \\
\hline Otra & 36 & 9,2 \\
\hline Básica & 41 & 10,6 \\
\hline Educación Superior & 115 & 29,6 \\
\hline Bachillerato & 161 & 41,5 \\
\hline Total & 388 & 100,0 \\
\hline
\end{tabular}




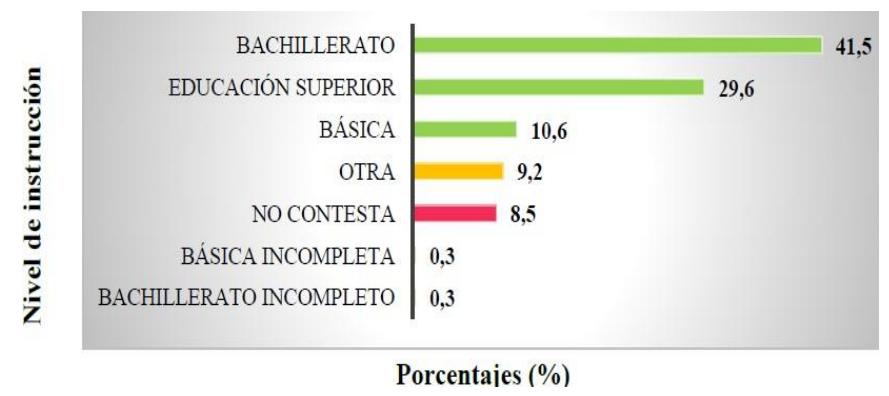

Fig. 2: Nivel de instrucción de los encuestados. Moreno E, 2017

Interpretación: En relación al nivel de instrucción, el 41,5\% de los encuestados cuenta con bachillerato terminado, el 29,6\% posee educación superior, el 10,6\% indica tener educación básica, el 9,2\% estudios de cuarto nivel, el 8,5\% no contestó, y el 0,3\% aseguró tener educación básica incompleta y un porcentaje similar bachillerato incompleto.

Pregunta 3: Parroquias urbanas de Riobamba

Tabla 3: Parroquias urbanas de Riobamba

\begin{tabular}{|l|l|l|}
\hline Parroquia & Frecuencia & Porcentaje \\
\hline No contesta & 12 & 3,1 \\
\hline Maldonado & 57 & 14,7 \\
\hline Yaruquíes & 63 & 16,2 \\
\hline Veloz & 83 & 21,4 \\
\hline Velasco & 85 & 21,9 \\
\hline Lizarzaburu & 88 & 22,7 \\
\hline Total & 388 & 100,0 \\
\hline
\end{tabular}

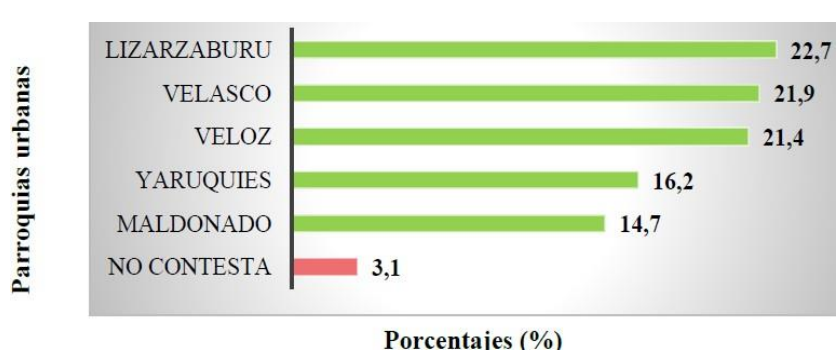

Fig.3: Parroquias urbanas. Moreno E, 2017 
Interpretación: La colaboración para con la encuesta en las parroquias urbanas es del 22,7\% en Lizarzaburu, seguidamente 21,9\% de Velasco, el 21,4\% de Veloz, el 16,2\% de Yaruquíes, el 14,7\% en Maldonado, y finalmente, un 3,1\% no contestó.

Pregunta 4: ¿Conoce los programas y proyectos que impulsa el Municipio de la ciudad de Riobamba por el bien común?

Tabla 4: Conocimiento sobre programas y proyectos

\begin{tabular}{|l|l|l|}
\hline $\begin{array}{l}\text { Conocimiento sobre } \\
\text { programas y proyectos }\end{array}$ & Frecuencia & Porcentaje \\
\hline No contesta & 1 & 0,3 \\
\hline Algunos & 80 & 20,6 \\
\hline $\mathrm{Si}$ & 147 & 37,9 \\
\hline No & 160 & 41,2 \\
\hline Total & 388 & 100,0 \\
\hline
\end{tabular}

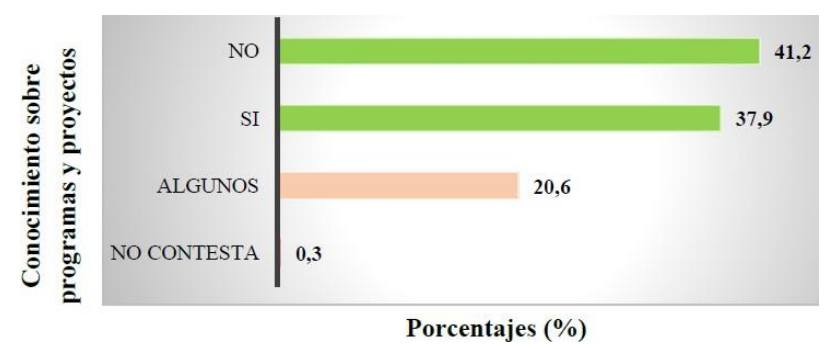

Fig.4: Conocimiento programas y proyectos. Moreno E, 2017

\section{Cuadro resumen}

Uno de los grandes retos de las políticas públicas, es equilibrar las desigualdades existentes con políticas públicas en el ámbito turístico que desarrollen oportunidades, y con más recursos de financiamiento bien administrados, que establezcan y alcancen objetivos comunes que sean alcanzables a largo plazo, políticas que tracen estrategias claras y que sean efectivas.

En el siguiente cuadro se muestra un listado de variables levantadas en el campo en las parroquias: Lizarzaburu, Maldonado, Veloz, Velasco y Yaruquíes.

Cabe indicar que para la elaboración de las fórmulas se necesita de un análisis matemáticoestadístico para analizar y obtener datos a profundidad sobre tendencias numéricas que ayuden a 
tomar decisiones más certeras, se aclara que este no es uno de los objetivos del presente trabajo. Es por eso que, el análisis de las variables para convertirlas en indicadores se realizará en una fase posterior del macro proyecto Riobamba sostenible GADM Riobamba-ESPOCH.

Tabla 31: Listado de variables levantadas en campo

\begin{tabular}{|c|c|c|c|c|}
\hline ÁMBITO & VARIABLES & INTERPRETACIÓN & FUENTE & RESULTADO \\
\hline \multirow[t]{6}{*}{$\begin{array}{l}\text { PARTICI- } \\
\text { PACIÓN } \\
\text { CIUDADANA }\end{array}$} & $\begin{array}{l}\text { 1. Porcentaje de personas } \\
\text { que indican que impulsa } \\
\text { programas y proyectos el } \\
\text { GADM Riobamba por el } \\
\text { bien común. }\end{array}$ & \multirow{2}{*}{$\begin{array}{lr}\text { Está destinado } & \text { a } \\
\text { determinar } & \text { el } \\
\text { porcentaje de interés y } & \\
\text { apego de la población } \\
\text { local sobre los } \\
\text { programas y proyectos } \\
\text { que impulsa el } \\
\text { municipio. }\end{array}$} & \multirow{2}{*}{\begin{tabular}{lr}
\multicolumn{3}{l}{ Elaboración con los } \\
técnicos de la \\
Dirección de Turismo \\
del $\quad$ Gobierno \\
Autónomo \\
Descentralizado \\
Municipal de \\
Riobamba y el equipo \\
técnico \\
investigación de \\
ESPOCH, 2017
\end{tabular}} & $37,9 \%$ \\
\hline & $\begin{array}{l}\text { 2. Porcentaje de personas } \\
\text { indicando que no impulsa } \\
\text { programas y proyectos el } \\
\text { Municipio el GADM } \\
\text { Riobamba por el bien } \\
\text { común }\end{array}$ & & & $41,2 \%$ \\
\hline & & \multirow{2}{*}{$\begin{array}{l}\text { Está destinado a } \\
\text { determinar el } \\
\text { porcentaje de interés y } \\
\text { apego por parte de los } \\
\text { establecimientos de } \\
\text { alojamiento y } \\
\text { gastronomía sobre los } \\
\text { programas y proyectos } \\
\text { que impulsa el } \\
\text { municipio }\end{array}$} & \multirow{2}{*}{\begin{tabular}{l}
\multicolumn{2}{l}{ Elaboración con los } \\
técnicos de la \\
Dirección de Turismo \\
del Gobierno \\
Autónomo \\
Descentralizado \\
Municipal de \\
Riobamba y el equipo \\
técnico r de \\
investigación r \\
ESPOCH, 2017
\end{tabular}} & $40 \%$ \\
\hline & $\begin{array}{l}4 . \quad \text { Porcentaje de } \\
\text { establecimientos de } \\
\text { alojamiento } \\
\text { gastronomía que indican } \\
\text { que no impulsa } \\
\text { programas y proyectos el } \\
\text { GADM Riobamba por el } \\
\text { bien común. }\end{array}$ & & & $58 \%$ \\
\hline & $\begin{array}{l}\text { 5. Porcentaje de personas } \\
\text { que mencionan la } \\
\text { existencia de un } \\
\text { asesoramiento sobre } \\
\text { políticas públicas por } \\
\text { parte del GADM } \\
\text { Riobamba a la ciudadanía } \\
\text { de las parroquiasurbanas. }\end{array}$ & \multirow[t]{2}{*}{ 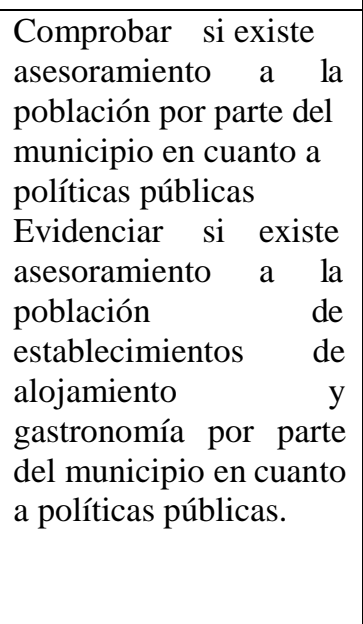 } & \multirow[t]{2}{*}{\begin{tabular}{l}
\multicolumn{2}{l}{ Elaboración con los } \\
técnicos de la \\
Dirección de Turismo \\
del Gobierno \\
Autónomo \\
Descentralizado \\
Municipal de \\
Riobamba y el equipo \\
técnico r de \\
investigación \\
ESPOCH, 2017
\end{tabular}} & $63,7 \%$ \\
\hline & $\begin{array}{l}\text { 6. Porcentaje de personas } \\
\text { que no tienen } \\
\text { conocimiento sobre el }\end{array}$ & & & $33,5 \%$ \\
\hline
\end{tabular}




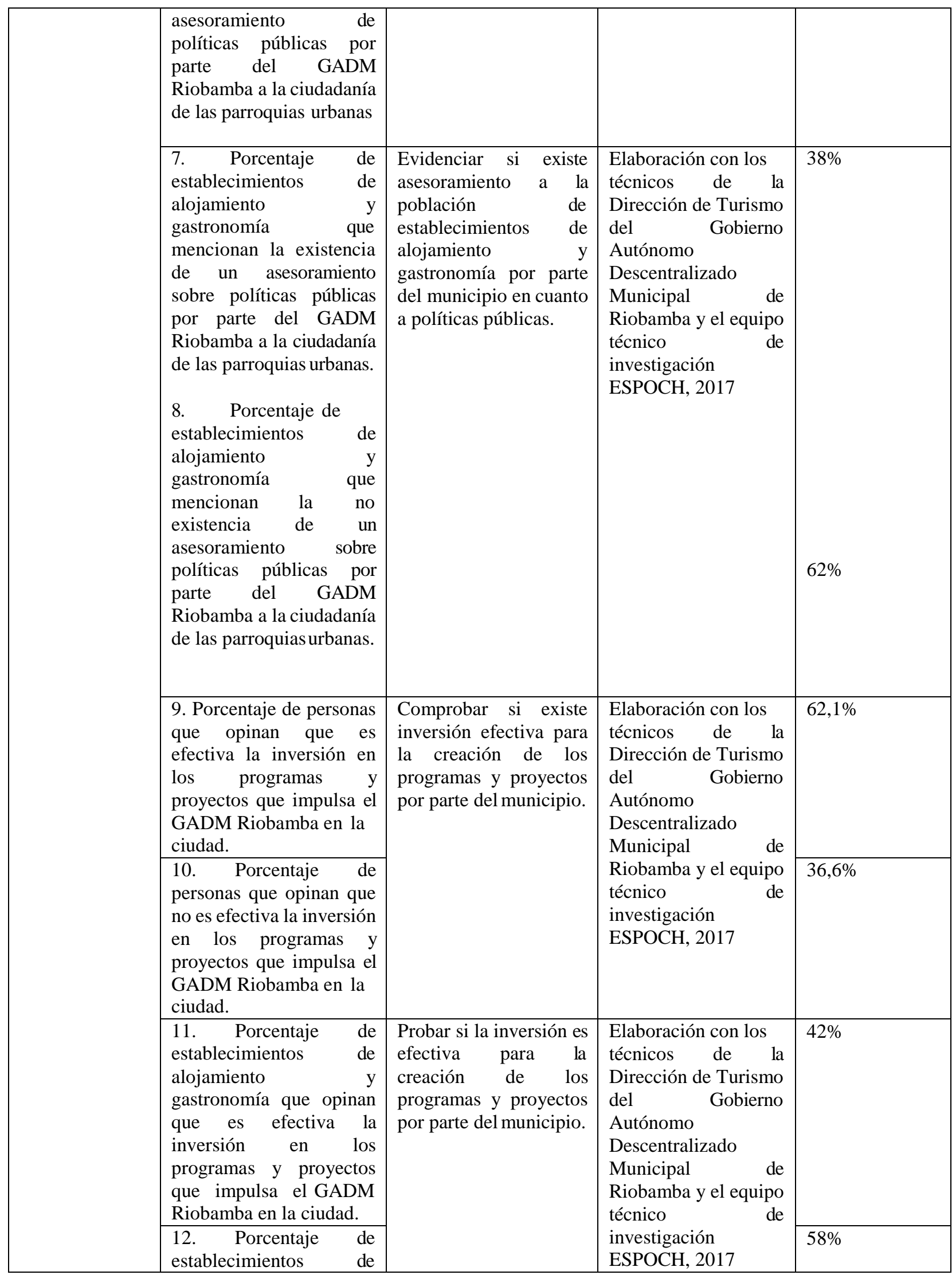




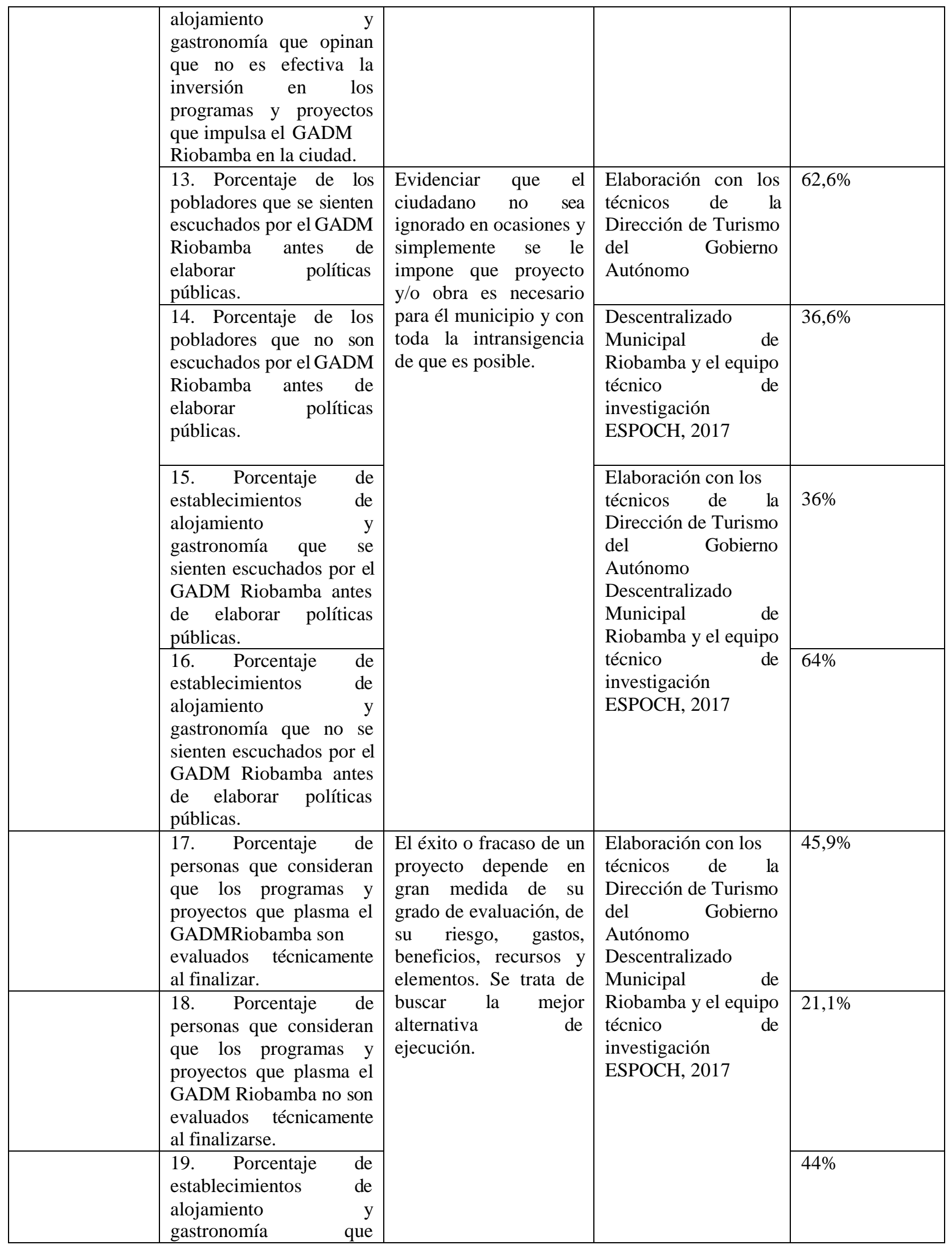




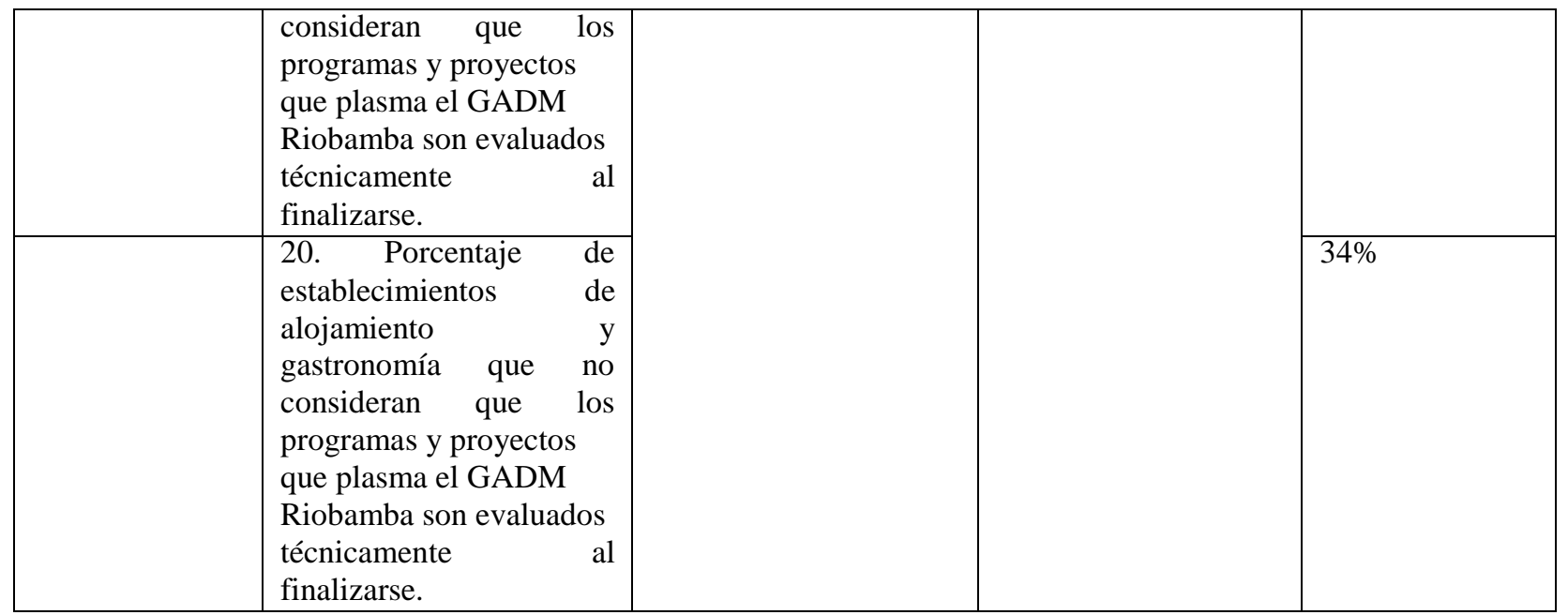

Realizado por: Moreno, E. 2017

\section{Incidencia de las variables recopiladas en el sector turístico de Riobamba.}

Para que exista un proceso de desarrollo local mediante las políticas públicas en al ámbito turístico, es preciso contar con las condiciones que garanticen la participación y la interacción de los diferentes actores implicados en este proceso. Además, la participación ciudadana es una instancia donde se puede observar, vigilar, dar seguimiento y evaluar; en el cual regularmente se propone mejoras e influir en las decisiones sobre el diseño y la aplicación de políticas públicas.

Para ello se requiere que los mandatarios en sus períodos correspondientes y la comunidad en general conozcan estas estrategias y se comprometan a continuar ininterrumpidamente a través del tiempo, en pro de mejorar las condiciones de vida y que midan impactos en la sociedad.

Se realizó una prueba de proporciones de una muestra con el fin de conocer la opinión de la ciudadanía y establecimientos de alojamiento y gastronomía sobre si el GADM Riobamba contribuye a potenciar el sector turismo de la ciudad.

A continuación, se presentan:

- Pregunta: Son variables recopiladas donde se analizó con el fin de saber si contribuyen o no al sector turístico.

- Resultados: Se aplicó la hipótesis planteada: donde se acepta o se rechaza la misma.

Ho: $\mathrm{p} \leq 0,4 \mathrm{Hi}: \mathrm{p} \geq 0,4$ 


\section{Pregunta 4}

¿Conoce los programas y proyectos que impulsa el Municipio de la ciudad de Riobamba por el bien común?

\section{Prueba de hipótesis*}

prop.test $(\mathrm{x}=147, \mathrm{n}=307, \mathrm{p}=0.40$, alternative $=$ "greater" $)=0.002881$

\section{**Resultado}

Como el p-value $=0.002881$ es menor que 0.05 entonces se rechaza la hipótesis nula, porque existe evidencia que la proporción de ciudadanos que tiene conocimiento sobre programas y proyectos que impulsa el Municipio de la ciudad de Riobamba por el bien común, es mayor 0.4 , por lo cual *potencia el sector turístico y la sostenibilidad integral en la ciudad de Riobamba al 95\% de confianza.

\section{Recomendación}

Se encuentra en el rango $\geq 0.4$ para potenciar la sostenibilidad, pero debe fortalecer aún más en este ámbito, realizando talleres para dar a conocer los programas y proyectos en el sector turístico que realiza el Municipio donde participe la ciudadanía.

\section{Pregunta 8}

¿Considera que el Municipio asesora sobre políticas públicas a la población?

\section{$\checkmark$ Prueba de hipótesis*}

prop.test $(\mathrm{x}=247, \mathrm{n}=377, \mathrm{p}=0.40$, alternative $=$ "greater" $)=2.2 \mathrm{e}-16$

\section{Resultado}

Como p-value $=2.2 \mathrm{e}-16$ es menor que 0.05 , entonces se rechaza la hipótesis nula, porque existe evidencia que la proporción de ciudadanos sobre el asesoramiento por parte del municipio sobre políticas públicas es mayor que 0.4 por lo cual potencia el sector turístico y la sostenibilidad integral en la ciudad de Riobamba al $95 \%$ de confianza.

\section{Recomendación}

Se encuentra en el en el rango $\geq 0.4$ para potenciar la sostenibilidad, aunque se debe informar sobre políticas públicas a la ciudadanía, una parte fundamental para el progreso de una ciudad. 


\section{Pregunta 9}

¿Cree Usted que el Municipio asiste técnicamente a pobladores al implementar políticas públicas?

\section{Prueba}

\section{Resultado}

Como p-value $=2.2 \mathrm{e}-16$ es menor 0.05 entonces se rechaza la hipótesis nula, porque existe evidencia que la proporción de ciudadanos sobre la asistencia técnica de la municipalidad sobre políticas públicas es mayor a 0.4 por lo cual potencia el sector turístico y la sostenibilidad integral en la ciudad de Riobamba al 95\% de confianza.

\section{Recomendación}

Se encuentra en el en el rango $\geq 0.4$ para potenciar la sostenibilidad, se debe capacitar a la ciudadanía sobre políticas públicas.

\section{Pregunta 10}

¿Considera que el Municipio de Riobamba escucha a los pobladores antes de elaborar políticas públicas?

\section{Prueba de hipótesis*}

prop.test $(\mathrm{x}=243, \mathrm{n}=385, \mathrm{p}=0.40$, alternative $=$ "greater" $)=2.2 \mathrm{e}-16$

\section{Resultado}

Como p-value $=2.2 \mathrm{e}-16$ es menor 0.05 entonces se rechaza la hipótesis nula, porque existe evidencia que la proporción de ciudadanos en la consulta sobre políticas públicas que realiza el municipio es mayor a 0.4 por lo cual potencia el sector turístico y la sostenibilidad integral en la ciudad de Riobamba al 95\% de confianza.

\section{Recomendación}

Se encuentra en el en el rango $\geq 0.4$ para potenciar la sostenibilidad, aunque se sugiere trabajar en conjuntamente con la ciudadanía

\section{Pregunta 11}

¿Usted opina que existe un monitoreo de las políticas públicas que impulsa el Municipio de Riobamba?

\section{Prueba de hipótesis*}


prop.test $(\mathrm{x}=185, \mathrm{n}=274, \mathrm{p}=0.40$, alternative $=$ "greater" $)=2.2 \mathrm{e}-16$

\section{Resultado}

Como p-value $=2.2 \mathrm{e}-16$ es menor que 0.05 entonces se rechaza la hipótesis nula, porque existe evidencia que la proporción de ciudadanos sobre la opinión del monitoreo por parte del municipio sobre políticas públicas es mayor a 0.4 por lo cual potencia el sector turístico y la sostenibilidad integral en la ciudad de Riobamba al $95 \%$ de confianza.

\section{Recomendación}

Se encuentra en el en el rango $\geq 0.4$ para potenciar la sostenibilidad. Además se debe dar seguimiento a la implementación de políticas públicas con el fin de contribuir a mejorar el trabajo.

\section{Pregunta 12}

¿Usted considera que los proyectos que realiza la Municipalidad de Riobamba son evaluados técnicamente al terminarse?

\section{Prueba de hipótesis*}

prop.test $(x=178, n=260, p=0.40$, alternative $=$ "greater" $)=2.2 \mathrm{e}-16$

\section{Resultado}

Como p-value $=2.2 \mathrm{e}-16$ menor que 0.05 entonces se rechaza la hipótesis nula, porque existe evidencia que la proporción de opiniones de los ciudadanos sobre la evaluación técnica realizada por el municipio sobre los proyectos es mayor a 0.4 por lo cual potencia el sector turístico y la sostenibilidad integral en la ciudad de Riobamba al $95 \%$ de confianza.

\section{Recomendación}

Se encuentra en el en el rango $\geq 0.4$ para potenciar la sostenibilidad. También continuar con la evaluación de las políticas públicas con el fin de mejorar este ámbito.

\section{Pregunta 9}

$¿$ Conoce los programas y proyectos que impulsa el Municipio de la ciudad de Riobamba por el bien común?

\section{Prueba de hipótesis*}

prop.test $(\mathrm{x}=20, \mathrm{n}=49, \mathrm{p}=0.40$, alternative $=$ "greater" $)=0.5$ 


\section{Resultado}

Como p-value $=0.5$ mayor que 0.05 entonces se la hipótesis nula se acepta, porque existe evidencia que la proporción de ciudadanos sobre el conocimiento de programas y proyectos impulsados por el municipio es menor a 0.4 por lo cual NO potencia el sector turístico y la sostenibilidad integral en la ciudad de Riobamba al $95 \%$ de confianza.

\section{Recomendación}

No se encuentra en el en el rango $\geq 0.4$ para potenciar la sostenibilidad. Por lo cual, debe realizar cambios en este ámbito para fortalecer al turismo.

\section{Pregunta 11}

¿Cree Usted que la inversión del Municipio de Riobamba en los programas y proyectos que impulsa es efectiva?

\section{Prueba de hipótesis*}

prop.test $(\mathrm{x}=21, \mathrm{n}=50, \mathrm{p}=0.40$, alternative $=$ "greater" $)=0.4426$

\section{Resultado}

Como p-value $=0.4426$ mayor que 0.05 entonces a hipótesis nula se acepta, porque existe evidencia que la proporción de opiniones de los ciudadanos sobre la inversión municipal en los programas y proyectos en menor a 0,4 por lo cual NO potencia el sector turístico y la sostenibilidad integral en la ciudad de Riobamba al $95 \%$ de confianza.

\section{Recomendación}

No se encuentra en el rango $\geq 0.4$ para potenciar la sostenibilidad. Por lo cual, debe realizar cambios en este ámbito para fortalecer al turismo existiendo una inversión equitativa por parte del Municipio hacia las políticas públicas.

\section{Pregunta 12}

¿Considera que el Municipio asesora sobre políticas públicas a la población?

\section{Prueba de hipótesis*}

prop.test $(\mathrm{x}=19, \mathrm{n}=50, \mathrm{p}=0.40$, alternative $=$ "greater" $)=0.5574$ 


\section{Resultado}

Como el p-value $=0.5574$ es mayor que 0.05 entonces la hipótesis nula se acepta, porque existe evidencia que la proporción de opiniones de los ciudadanos sobre el asesoramiento sobre políticas públicas por parte del municipio es menor que 0.4 por lo que NO potencia el sector turístico y la sostenibilidad integral en la ciudad de Riobamba al 95\% de confianza

\section{Recomendación}

No se encuentra en el en el rango $\geq 0.4$ para potenciar la sostenibilidad. Por lo cual, debe realizar cambios en este ámbito para fortalecer al turismo, existiendo un asesoramiento por parte del Municipio a la ciudadanía sobre las políticas públicas.

\section{Pregunta 13}

¿Cree Usted que el Municipio asiste técnicamente a pobladores al implementar políticas públicas en las áreas de hotelería y gastronomía?

\section{Prueba de hipótesis*}

prop.test $(x=20, n=50, p=0.40$, alternative $=$ "greater" $)=0.5$

\section{Resultado}

Como p-value $=0.5$ mayor que 0.05 entonces la hipótesis nula se acepta, porque existe evidencia que la proporción de opiniones de los ciudadanos sobre la asistencia técnica que realiza el municipio es menor a 0.4 por lo que NO potencia el sector turístico y la sostenibilidad integral en la ciudad de Riobamba al 95\% de confianza.

\section{Recomendación}

No se encuentra en el rango $\geq 0.4$ para potenciar la sostenibilidad. Por lo cual, debe realizar cambios en este ámbito para fortalecer al turismo con la ayuda de charlas, talleres por parte del Municipio a la ciudadanía sobre las políticas públicas.

\section{Pregunta 14}

¿Considera que el Municipio de Riobamba escucha a los pobladores antes de elaborar políticas públicas?

\section{Prueba de hipótesis*}

prop.test $(\mathrm{x}=18, \mathrm{n}=50, \mathrm{p}=0.40$, alternative $=$ "greater" $)=0.6675$ 


\section{Resultado}

Como p-value $=0.6675$ mayor que 0.05 entonces la hipótesis nula se acepta, porque existe evidencia que la proporción de ciudadanos sobre la comunicación que existe por parte del municipio con los pobladores es menor a 0.4 por lo cual no potencia el sector turístico y la sostenibilidad integral en la ciudad de Riobamba al 95\% de confianza.

\section{Recomendación}

No se encuentra en el en el rango $\geq 0.4$ para potenciar la sostenibilidad. Por lo cual, debe realizar cambios en este ámbito para fortalecer al turismo con la ayuda de existencia de comunicación en todo momento por parte del Municipio a la ciudadanía sobre las políticas públicas.

\section{Pregunta 15}

¿Usted opina que existe un monitoreo de las políticas públicas que impulsa el Municipio de Riobamba?

\section{Prueba de hipótesis*}

prop.test $(\mathrm{x}=17, \mathrm{n}=38, \mathrm{p}=0.40$, alternative $=$ "greater" $)=0.3334$

\section{Resultado}

Como $\mathrm{p}$-value $=0.3334$ mayor que 0.05 entonces la hipótesis nula se acepta, porque existe evidencia que la proporción de opiniones de los ciudadanos sobre el monitoreo que realiza la municipalidad a las políticas públicas es menor a 0.4 por lo cual no potencia el sector turístico y la sostenibilidad integral en la ciudad de Riobamba al $95 \%$ de confianza.

\section{Recomendación}

No se encuentra en el en el rango $\geq 0.4$ para potenciar la sostenibilidad. Por lo cual, debe realizar cambios en este ámbito para fortalecer al turismo.

\section{Pregunta 24}

¿Se siente beneficiado por el Municipio en las actividades comerciales que realiza?

\section{Prueba de hipótesis*}

prop.test $(\mathrm{x}=16, \mathrm{n}=39, \mathrm{p}=0.40$, alternative $=$ "greater" $)=0.5$ 


\section{Resultado}

Como el p-value $=0.5$ y este es mayor que 0.05 entonces la hipótesis nula se acepta, porque existe evidencia que la proporción de ciudadanos que se sienten beneficiados por parte del municipio en las actividades comerciales es menor a 0.4 por lo cual no potencia el sector turístico y la sostenibilidad integral en la ciudad de Riobamba al $95 \%$ de confianza.

\section{Recomendación}

No se encuentra en el en el rango $\geq 0.4$ para potenciar la sostenibilidad. Por lo cual, debe realizar cambios en este ámbito para fortalecer al turismo.

\section{Pregunta 25}

¿Considera que el Municipio de la Ciudad promueve la actividad turística a través de impulsar el comercio gastronómico y hotelero?

\section{Prueba de hipótesis*}

prop.test $(\mathrm{x}=14, \mathrm{n}=50, \mathrm{p}=0.40$, alternative $=$ "greater" $)=0.5$

\section{Resultado}

Como el p-value $=0.5$ y este es mayor que 0.05 entonces la hipótesis nula se acepta, porque existe evidencia que la proporción de opiniones de los ciudadanos sobre la promoción de la actividad gastronómica y hotelera por parte del municipio es menor a 0.4 por lo cual no potencia el sector turístico y la sostenibilidad integral en la ciudad de Riobamba al $95 \%$ de confianza.

\section{Recomendación}

No se encuentra en el en el rango $\geq 0.4$ para potenciar la sostenibilidad. Por lo cual, debe realizar cambios en este ámbito para fortalecer al turismo.

\section{Nota:}

No potencia al sector turístico

Potencia al sector turístico

*Potenciar el turismo: Fomentar, impulsar, promover el turismo **Resultado 
H0: $p=0.5$ (Proporción de opiniones afirmativas en la población es el 50\%)

H1: $p \neq 0.5$ (Proporción de opiniones afirmativas en la población no es el 50\%)

Nivel de significancia $\alpha=0.05$

Criterio: Rechazar la hipótesis nula si p-valor $>0.05$, donde el estadístico es $Z 0$

Nivel de confianza del $95 \%$

Toda la información presentada ha sido resumida y compilada en un boletín informativo que se encuentra en revisión por parte del equipo de investigadores de la ESPOCH y los técnicos de turismo del GADM Riobamba; el mismo que pretende ser difundido en las dos audiencias de interés estudiadas: ciudadanía y prestadores de servicios hoteleros de la ciudad.

\section{Conclusiones}

Según los resultados obtenidos en el análisis de las encuestas en cuanto a políticas públicas por el bien común, se pudo apreciar lo siguiente: el 41,2\% menciona que el GADM Riobamba no impulsa programas y proyectos; el 63,7\% señala que existe un asesoramiento sobre políticas; el $62,1 \%$ consideran que es efectiva la inversión que realiza el municipio; el 62,6\% son escuchados por el GADM, el 45,9\% afirman que son evaluados al finalizar; el 55,4\% conocen las políticas turísticas que impulsa el GADM; Por el contrario en cuanto a las encuestas realizadas en los establecimientos de alojamiento y gastronomía señalan que el $66 \%$ no se sienten beneficiados por las actividades que realiza el GADM, el 78\% señala que no existe promoción alguna sobre el turismo de la ciudad.

En base a la información obtenida, con un $95 \%$ de confianza se pudo probar que el Gobierno Autónomo Descentralizado Municipal de Riobamba brinda: asistencia técnica, asesoramiento, monitoreo y evaluación técnica a la ciudadanía de las parroquias urbanas de Riobamba.

Mediante la investigación sobre indicadores políticos en el ámbito turístico, sintetizando los que se adapten al territorio de estudio agrupado por ámbitos, fueron: socio-cultural con 3 indicadores, gestión y participación ciudadana con 9 indicadores, económica con 1 indicador. Los cuales fueron tomados como sugerencia para el GADM Riobamba para una fase posterior de estudio.

El boletín informativo un medio de difusión donde se puede obtener información relevante, el mismo que hace referencia a las potencialidades y debilidades que tiene la ciudad de Riobamba en 
cuanto a lo político en el ámbito turístico, misma que servirá como instrumento para dar a conocer a la ciudadanía.

\section{Referencias Bibliográficas}

Asamblea Nacional del Ecuador. (2008). Título V Organización Territorial del Estado Ecuatoriano. Competencias de los GADS. Recuperado el 20 de octubre de 2017, en: http://www.efemerides.ec/1/cons/index5.htm.

Armijo, M. (Junio 2011). Planificación estratégica e indicadores de desempeño. Comisión Económica para América Latina y el Caribe (CEPAL). Santiago de Chile. p. 26.

Aguilar Villanueva, Luis. (Agosto 2006). Gobernanza y gestión pública. México: FCE. p. 18

Arciniegas, M. (2011). Fundamentos de los Sistemas de Información Geográfica. Recuperado el 11 de octubre de 2017, en: http://www.iadb.org/en/topics/urbandevelopment/housing,2850.html Ávila, H. (Enero 2006). Introducción a la metodología de la investigación. Estudio encuesta. Edición electrónica. Recuperado el 21 de agosto del 2017, en: https://www.uam.es/personal_pdi/stmaria/jmurillo/InvestigacionEE/Presentaciones/Curso_10/EN CUESTA_1_Trabajo.pdf

Banco Interamericano de Desarrollo. BID. (2014). Estudio teórico y empírico del impacto de los procesos de reforma política en GRD. Washington DC. EEUU. p. 200

Borroso, M., \& Flores, D. (Julio 2007) La política turística como parte de la política económica. Análisis Turístico. pp. 5-21

Briones, G. (2002). Programa de especialización en teoría, métodos y técnicas de investigación social. Metodología de la investigación cuantitativa en las cienias sociales en las ciencias sociales. p. 196. Bogota - Colombia. Recuperado el 22 de Agosto de 2017, en: https://es.slideshare.net/DrRebilla/metodologia-de-la-investigacion-guillermo-briones

Cepeda, F. (2012). Riobamba imagen, palabra e historia. Riobamba: Casa de la Cultura Ecuatoriana Benjamín Carrión, Núcleo de Chimborazo. Recuperado el 02 de agosto de 2017 en: http://revistaprocesos.ec/ojs/index.php/ojs/article/view/111 
Centro de Políticas Públicas UC (2015). Confianza, la clave para el desarrollo de Chile. Santiago: Centro de Políticas Públicas UC. p. 150

Comisión Económica para América Latina y el Caribe. (2010). La hora de la igualdad. Cap. V: "El lugar importa: disparidades y convergencias territoriales”. Santiago de Chile. p. 157.

Delgado, L. (2009). Las políticas públicas: ciclo de las políticas públicas, clases de políticas públicas, eficacia, legalidad y control. Indicadores de gestión. Documentación sobre gerencia pública, del subgrupo A2, cuerpo técnico, especialidad de gestión administrativa, de la administración de la junta de comunidades de Castilla-La Mancha. Tema 3. Consejería de administraciones públicas. Escuela de administración regional, 95.

Departamento Administrativo Nacional de Estadística. (2002). Elementos metodológicos básicos para la selección, construcción, interpretación y análisis de indicadores. SNIE. Bogotá. p. 42.

Díaz, M. (3 de Junio de 2013). Características de un boletín informativo. Recuperado el 25 de Abril de 2017, en: http://marianadiaz028.blogspot.com/2013/06/concepto-y-caracteristicas-de-un.html

Equipo Consultor Tourism \& Leisure - Europraxis. (2007). Diseño del plan estratégico de desarrollo de turismo sostenible para ecuador "plandetur 2020". Ecuador. Recuperado el 18 de Agosto del 2017, en: http://www.turismo.gob.ec/wpcontent/uploads/downloads/2013/02/PLANDETUR-2020.pdf.

Fernández, F. (2006). Indicadores de sostenibilidad y medio ambiente: métodos y escala. Sevilla: Dirección General de Educación Ambiental y Sostenibilidad. Recuperado el 19 de agosto del 2017. en:

https://www.juntadeandalucia.es/medioambiente/web/Bloques_Tematicos/Sostenibilidad/Estrate gia_andaluza_desarrollo_sostenible/libro_indicadores_sostenibilidad/1_portada_e_indice.pdf. Págs. 24.

Ferrer, J. (20 de Enero de 2011). La TV quedó relegada por internet en los EEUU, Europa y China. Recuperado el 26 de Agosto de 2017, en INFORMADOR. MX: http://www.informador.com.mx/tecnologia/2011/265055/6/internet-es-el-medio-decomunicacion-mas-usado-en-eu-europa-y-china.htm 
Gallopín, G. (2006). Indicadores de sostenibilidad, aspectos conceptuales y metodológicos. pp. 810. Recuperado el 14 de Mayo de 2017, en: ftp://ftp.utalca.cl/redcauquenes/cauquenes\%20estudio/Articulos/Gallopin_LOS\%20indicadores\% 20DE\%20desarrollo\%20sustentable.\%20aspectos\%20conceptuales\%20Y\%20metodologicos.pdf

García, A. G. (2014). Indicadores de sostenibilidad turística. Recuperado el 18 de Agosto de 2017, en:http:/fcetou.uvigo.es/files/docencia/TFG/premios/Indicadores\%20de\%20sostenibilidad\%20tu ristica.pdf

Instituto Nacional de Estadísticas y Censos. (2010). Censo de población y vivienda. Quito: INEC. Ivars, J. (2006). La política turística. En Sáez, A.; Matín, P. y pulido, J.L. (Ed.); Estructura económica del turismo. Síntesis. Madrid. pp. 261-284.

Jimenez, A. (Octubre de 2013). Estadística: representación tabular y gráfica de datos. Recuperado el 28 de agosto de 2017, en: http://estadisticacrisanto.blogspot.com/2013/10/representaciontabular-y-grafica-de.html

Kuby, P., \& Robert, J., (2005). Estadística elemental, lo esencial (3ª ed). Thomson. ISBN 970-686287-0. Recuperado el 29 de marzo del 2017, en: http://csonkametodosytecnicasdeinvestigacin.blogspot.com/2011/09/metodos-de-recoleccion-de-datos-enla.html

Maggiolo, I., \& Perozo, J. (2007). Políticas públicas: proceso de concertación Estado-Sociedad. Revista Venezolana de Gerencia. 12(39); 392.

Menendez, J. (26 de Junio de 2014). Municipio y bomberos de Quito buscan reducir incendios con el plan fuego. Recuperado el 01 de Septiembre de 2017, en: http://www.ecuavisa.com/articulo/noticias/nacional/69257-municipio-bomberos-quito-buscanreducir-incendios-plan-fuego

Michelini. (2013). Introducción-boletin informativo. Recuperado el 11 de octubre de 2017, en: http://www.iadb.org/en/topics/urban-development/housing,2850.html

Ortiz, F. (2005). Metodología de la Investigación. México: Limusa. p. 180. 
Pérez, A. (2002). ¿Qué son los indicadores? Revsita de información y análisis num. 19. Recuperado el 18 de agosto de 2017, en http://www.orion2020.org/archivo/sistema_mec/10_indicadores2.pdf

Pino, R., \& Díaz, J. (Febrero de 2011). Lecciones aprendidas desde la experiencia española de análisis de la percepción ciudadana de los servicios públicos. CLAD Reforma y Democracia. No. 49., p. 15.

Riola, R. (2005). Modelo Estadístico. Recuperado 11 de octubre de 2017, de la Escuela de Andaluza de Salud Pública: http://www.divestadistica.es/es/que_es_un_modelo_estadistico.html.

Rivas, J., \& Magadán, M. (2007). Los indicadores de sostenibilidad en el turismo. Revista de Economía, Sociedad, Turismo y Medio Ambiente - RESTMA, (6), p. 30

Torres, R. (15 de Diciembre de 2014). Participación ciudadana en la formulación de las políticas públicas locales, el caso del distrito vi del municipio de Managua, (2007-2013). Recuperado el 19 de agosto de 2017 de Universidad de Chile Facultad de Ciencias Físicas y Matemáticas Departamento de Ingeniería Industrial: Recuperado el 20 de diciembre del 2017, en:

.http://repositorio.uchile.cl/bitstream/handle/2250/131430/Participacion-ciudadana-en-laformulacion-de-politicas-publicas... pdf?sequence $=1$

Urquizo, A. (2013). Matemática dirigida a otras ciencias. Riobamba: Instituto de Postgrado y Educación Continua (IPEC). Recuperado el 13 de Septiembre de 2017 en: http://dspace.espoch.edu.ec/bitstream/123456789/4442/1/20T00651.pdf

Vargas, M. (2009). Democracia deliberativa y gobernanza en el gobierno local: Análisis y evaluación de mecanismos institucionales de participación ciudadana en tres ciudades medias de Mexico. Universidad Nacional Autónoma de México. Recuperado el 10 de octubre en: <uaslp.academia.edu/marcoivanvargas/Papers>[Acceso 10 de octubre, 2014].

Velasco, M. (2016). Entre el poder y la racionalidad: gobierno. Turismo y Patrimonio Cultural. ISSN 1695-7121, Vol. 14, p. 577-594. Quito

Vera, J., (Dir.), Juárez, C., Morte, A., Torres, F., Navalón, M., Such, M., \& Ivars, J. (2001). Planificación y gestión del desarrollo turístico sostenible: propuestas para la creación de un sistema de indicadores. Alicante: Instituto Universitario de Geografía, Universidad de Alicante. p. 150 
